\title{
Asymptotic Behavior of Solutions for Nonlinear Volterra Discrete Equations
}

\author{
E. Messina, ${ }^{1}$ Y. Muroya, ${ }^{2}$ E. Russo, ${ }^{1}$ and A. Vecchio $^{3}$ \\ ${ }^{1}$ Dipartimento di Matematica e Applicazioni, Università degli Studi di Napoli "Federico II", \\ Complesso Monte S. Angelo, Via Cintia, 80126 Napoli, Italy \\ ${ }^{2}$ Department of Mathematics, Waseda University, 3-4-1 Ohkubo Shinjuku-ku, Tokyo 169-8555, Japan \\ ${ }^{3}$ Istituto per le Applicazioni del Calcolo "Mauro Picone", Sede di Napoli, Via P. Castellino 111, \\ 80131 Napoli, Italy
}

Correspondence should be addressed to A. Vecchio, vecchio@na.iac.cnr.it

Received 19 June 2007; Revised 14 January 2008; Accepted 5 May 2008

Recommended by A. Matsumoto

We consider nonlinear difference equations of the unbounded order of the form $x_{i}=b_{i}-$ $\sum_{j=0}^{i} a_{i, j} f_{i-j}\left(x_{j}\right), i=0,1,2, \ldots$, where $f_{j}(x)(j=0, \ldots, i)$ are suitable functions. We establish sufficient conditions for the boundedness and the convergence of $x_{i}$ as $i \rightarrow+\infty$. Some of these conditions are interesting mainly for studying stability of numerical methods for Volterra integral equations.

Copyright $@ 2008$ E. Messina et al. This is an open access article distributed under the Creative Commons Attribution License, which permits unrestricted use, distribution, and reproduction in any medium, provided the original work is properly cited.

\section{Introduction}

We consider the following nonlinear Volterra discrete equation of nonconvolution type:

$$
\begin{aligned}
& x_{i}=b_{i}-\sum_{j=0}^{i} a_{i, j} f_{i-j}\left(x_{j}\right), \quad i=0,1,2, \ldots, \\
& x_{i}, b_{i}, a_{i, j} \in I R, \quad b_{i} \neq 0, \forall i=0,1,2, \ldots
\end{aligned}
$$

The existence problem for solution of Volterra discrete equations arises in the nonlinear implicit case. For linear implicit equations and nonlinear explicit equations, the problem is easily solved. Recently, some local and global existence theorems for Volterra discrete equations in the general case are given in $[1,2]$. 
From now on, we assume that there exists a strictly increasing function $f(x)$ such that

$$
\begin{aligned}
& 0 \leq f_{i}(x) \leq f(x) \quad \text { for } x \geq 0, \\
& 0 \geq f_{i}(x) \geq f(x) \quad \text { for } x \leq 0,
\end{aligned} \quad i \geq 0 .
$$

Note that (1.2) implies that

$$
f(0)=0, \quad f_{i}(0)=0, \quad i \geq 0 .
$$

The above difference equation can be considered as the discrete counterpart of the Volterra integral equation whose importance in the applications is well known (see, e.g., $[3,4])$, and arises also in the application of numerical methods to Volterra integral and integrodifferential equations. The theory of the qualitative behavior of this type of nonlinear difference equation is very important, in particular for the study of numerical stability of such methods (see, e.g., [5-11] and the references therein).

In this paper, we study some sufficient conditions for the boundedness of the solutions (if they exist) of (1.1), subject to (1.2), and their asymptotic behavior as $i \rightarrow+\infty$. In particular, in Section 2 we investigate the asymptotic behavior when $\left|f_{i}(x)\right|$ is upper bounded by a linear function. The case of nonnegative coefficients is investigated in Section 3 and, with additional monotonicity assumptions, in Section 4.

2. Case of $\left|f_{i}(x)\right| \leq|x|, i \geq 0$

Assume that, in (1.1) with (1.2), the following additional hypotheses hold:

$$
\inf _{i \geq 0} a_{i, i}>-1, \quad\left|f_{i}(x)\right| \leq|x|, \quad \text { for any } x \in(-\infty,+\infty), i \geq 0 .
$$

Observe that the second part of (2.1) is true if in (1.2) $f(x)=x$. The following lemma can be easily proved.

Lemma 2.1. If

$$
\begin{aligned}
& a_{i i} \geq 0, \quad \text { then }|x| \leq\left|x+a_{i i} f_{0}(x)\right|, \\
& a_{i i} \leq 0, \quad \text { then }\left(1+a_{i i}\right)|x| \leq\left|x+a_{i i} f_{0}(x)\right|,
\end{aligned}
$$

for all $x \in \mathbb{R}$.

Here and in the sequel we assume a sum with a negative superscript to be zero. By using (2.1) and Lemma 2.1, from (1.1), we have that

$$
\left|x_{i}\right| \leq\left|\tilde{b}_{i}\right|+\sum_{j=0}^{i-1}\left|\tilde{a}_{i, j}\right|\left|x_{j}\right|, \quad i \geq 0,
$$

and we set

$$
\tilde{b}_{i}=\frac{b_{i}}{1+\min \left(0, a_{i, i}\right)}, \quad \tilde{a}_{i, j}=\frac{a_{i, j}}{1+\min \left(0, a_{i, i}\right)}, \quad 0 \leq j \leq i-1 .
$$

This inequality will be useful in order to find sufficient conditions for the boundedness of $x_{i}$ and for its convergence to zero as $i$ tends to infinity. 
Theorem 2.2. Consider (1.1) with (1.2) and (2.1), if there exists a positive constant A such that

$$
\begin{gathered}
\sup _{0 \leq j \leq i-1}\left|\tilde{a}_{i, j}\right| \leq A<+\infty, \quad \forall i \geq 1, \\
B=\sup _{i \geq 0}\left|\tilde{b}_{i}\right|<+\infty, \quad \bar{A}_{0}=\sup _{i \geq i_{0}} \sum_{j=i_{0}}^{i-1}\left|\tilde{a}_{i, j}\right|<1
\end{gathered}
$$

for some positive integer $i_{0}$, then $x_{i}$ is bounded and

$$
\left|x_{i}\right| \leq \frac{(1+A)^{i_{0}} B}{1-\bar{A}_{0}}<+\infty, \quad i \geq i_{0}
$$

Moreover, if

$$
\lim _{i \rightarrow \infty} b_{i}=0, \quad \lim _{i \rightarrow \infty}\left|a_{i, j}\right|=0 \quad \forall j \geq 0,
$$

then $\lim _{i \rightarrow \infty} x_{i}=0$.

Proof. Let us consider (2.3), by using (2.5), we have that

$$
\begin{aligned}
& \left|x_{0}\right| \leq\left|\tilde{b}_{0}\right| \leq B \\
& \left|x_{1}\right| \leq\left|\widetilde{b}_{1}\right|+\left|\tilde{a}_{1,0}\right|\left|x_{0}\right| \leq B+A B=(1+A) B \\
& \left|x_{2}\right| \leq\left|\tilde{b}_{2}\right|+\left|\tilde{a}_{2,0}\right|\left|x_{0}\right|+\left|\tilde{a}_{2,1}\right|\left|x_{1}\right| \leq B+A B+A(1+A) B=(1+A)^{2} B, \ldots, \\
& \left|x_{i}\right| \leq\left|\tilde{b}_{i}\right|+\sum_{j=0}^{i-1}\left|\tilde{a}_{i, j}\right|\left|x_{j}\right| \leq B+\sum_{j=0}^{i-1} A\left\{(1+A)^{j} B\right\}=B+\left\{(1+A)^{i}-1\right\} B=(1+A)^{i} B, \quad i \geq 0 .
\end{aligned}
$$

In particular, assume that the third part of (2.5) holds, then

$$
\left|x_{i}\right| \leq\left\{\left|\tilde{b}_{i}\right|+\sum_{j=0}^{i_{0}-1}\left|\tilde{a}_{i, j}\right|\left|x_{j}\right|\right\}+\sum_{j=i_{0}}^{i-1}\left|\tilde{a}_{i, j}\right|\left|x_{j}\right|, \quad \forall i \geq i_{0},
$$

and thus,

$$
\left|x_{i}\right| \leq\left\{\left|\tilde{b}_{i}\right|+\sum_{j=0}^{i_{0}-1}\left|\tilde{a}_{i, j}\right|\left|x_{j}\right|\right\}+\bar{A}_{0_{0} \leq j \leq i} \max _{i_{j}}\left|x_{j}\right| .
$$

Hence, the following inequalities hold for each $k \leq i$ :

$$
\left|x_{k}\right| \leq\left\{\left|\tilde{b}_{i}\right|+\sum_{j=0}^{i_{0}-1}\left|\tilde{a}_{i, j}\right|\left|x_{j}\right|\right\}+\bar{A}_{0} \max _{i_{0} \leq j \leq k}\left|x_{j}\right| \leq\left\{\left|\tilde{b}_{i}\right|+\sum_{j=0}^{i_{0}-1}\left|\tilde{a}_{i, j}\right|\left|x_{j}\right|\right\}+\bar{A}_{0} \max _{i_{0} \leq j \leq i}\left|x_{j}\right| .
$$

For this reason,

$$
\max _{i_{0} \leq j \leq i}\left|x_{j}\right| \leq\left\{\left|\tilde{b}_{i}\right|+\sum_{j=0}^{i_{0}-1}\left|\tilde{a}_{i, j}\right|\left|x_{j}\right|\right\}+\bar{A}_{0} \max _{i_{0} \leq j \leq i}\left|x_{j}\right|
$$


from which we obtain that

$$
\max _{i_{0} \leq j \leq i}\left|x_{j}\right| \leq \frac{\left\{B+\sum_{j=0}^{i_{0}-1} A(1+A)^{j} B\right\}}{1-\bar{A}_{0}}=\frac{(1+A)^{i_{0}} B}{1-\bar{A}_{0}}<+\infty, \quad i \geq i_{0} .
$$

Thus, $\left|x_{i}\right|$ is bounded and satisfies (2.6).

Assume that $\bar{x}=\lim \sup _{i \rightarrow \infty}\left|x_{i}\right|>0$ and put $\bar{r}=\sup _{i \geq i_{0}} \sum_{j=i_{0}}^{i-1}\left|a_{i, j}\right|, \gamma=1+\min \left(0, \inf _{i \geq i_{0}} a_{i, i}\right)$ and $M=\sup _{i \geq 0}\left|x_{i}\right|$. Then, since $\left|x_{i}\right|$ is bounded and the third of (2.5) holds, we have that $M<$ $+\infty$ and $\bar{r}<\gamma$. Let's take any $\epsilon>0$ and consider a continuous function $F(x)=(\bar{r}+x)(\bar{x}+x)+2 x$ on $[0, \epsilon]$. Then, by $F(0)=\bar{r} \bar{x}<\gamma \bar{x}$, there exists a constant $0<\epsilon_{0}<\epsilon$ such that

$$
F\left(\epsilon_{0}\right)=\left(\bar{r}+\epsilon_{0}\right)\left(\bar{x}+\epsilon_{0}\right)+2 \epsilon_{0}<\gamma \bar{x} .
$$

For the above $\epsilon_{0}>0$, there exists a positive integer $i_{1} \geq i_{0}$ such that $\left|x_{i}\right|<\bar{x}+\epsilon_{0}$ and $\sum_{j=i_{0}}^{i-1}\left|a_{i, j}\right|<$ $\bar{r}+\epsilon_{0}$, for any $i \geq i_{1}$. By assumption (2.7), we have that for $\epsilon_{1}=\epsilon_{0} /(1+M)>0$, there exists a positive integer $i_{2} \geq i_{1}$ such that

$$
\left|b_{i}\right|<\epsilon_{1}, \quad \sum_{j=0}^{i_{2}-1}\left|a_{i, j}\right|<\epsilon_{1} \quad \text { for any } i \geq i_{2}
$$

Then, for $i \geq i_{2}$ and $\tilde{c}_{i}=b_{i}-\sum_{j=0}^{i_{2}-1} a_{i, j} f_{i-j}\left(x_{j}\right)$, we have that

$$
\begin{gathered}
\left|\widetilde{c}_{i}\right| \leq\left|b_{i}\right|+\left(\sum_{j=0}^{i_{2}-1}\left|a_{i, j}\right|\right) M<\epsilon_{1}+\epsilon_{1} M=\epsilon_{0}, \\
\left|x_{i}\right| \leq \sum_{j=i_{2}}^{i-1}\left|a_{i, j}\right|\left|f_{i-j}\left(x_{j}\right)\right|+\left|\widetilde{c}_{i}\right|<\left(\bar{r}+\epsilon_{0}\right)\left(\bar{x}+\epsilon_{0}\right)+\epsilon_{0}<\bar{x}-\epsilon_{0}, \quad i \geq i_{2},
\end{gathered}
$$

which is a contradiction with the limsup definition. Hence, $\bar{x}=0$ and we obtain $\lim _{i \rightarrow \infty} x_{i}$ $=0$.

Note that the third part of (2.5) is equivalent to $\bar{r}=\sup _{i \geq i_{0}} \sum_{j=i_{0}}^{i-1}\left|a_{i, j}\right|<\gamma=1+\min (0$, $\left.\inf _{i \geq i_{0}} a_{i, i}\right)$.

The theorem above gives some conditions on the coefficients $a_{i j}$ of (1.1) for the boundedness of $x_{i}$ which supplement the results in [12, Theorem 2.1]. Moreover, it worths while to compare our result with the ones in [9, Theorem 3.1] and [5, Theorem 4.1]. In order to do that, we assume $a_{i i}=0, b_{i}=0$ and then $x_{0}$ is given. In this case, following the line of the proof of Theorem 2.2, we can still show that $x_{i}$ vanishes as $i \rightarrow \infty$ provided that (2.5) and the second part of (2.7) hold. Observe that this represents an additional result with respect to [9, Theorem 3.1] and [5, Theorem 4.1] which, involving the sum of the coefficients $\tilde{a}_{i, j}$ on the second index, enlarges the set of conditions for $x_{i}$ to be bounded and convergent to zero. As an example, for equation

$$
x_{i}=\sum_{j=0}^{i-1} \frac{1}{(i+1) 2^{i-j}} x_{j}
$$


(3.2) in [9] or the sufficient condition in [5] is not satisfied, however (2.5) is fulfilled. Moreover, it is easy to see that, in the convolution case $a_{i, j}=a_{i-j}$, the third of (2.5) coincides with the known one $[5,10]$

$$
\sum_{l=0}^{+\infty}\left|a_{l}\right|<1
$$

and the second part of (2.7) is implied by (2.5).

Theorem 2.2 turns out to be quite useful in the linear case when (1.1) represents the linearized equation for the global error of a numerical method applied to a Volterra integral equation. In this case, $b_{i}$ represents the local truncation error of the method at the step $i$. Thus, if $b_{i}$ is bounded for all $i$ and if (2.5) holds, then the error $x_{i}$ is bounded and the bound is given in (2.6).

The following theorem provides some sufficient conditions on the coefficients of (1.1) for the summability of $\left\{x_{i}\right\}_{i=0}^{+\infty}$, which turn out to be less restrictive of those stated by [13, Theorem 2.8].

Theorem 2.3. For (1.1) with (1.2), assume (2.1). If

$$
\bar{B}=\sum_{i=0}^{\infty}\left|\widetilde{b}_{i}\right|<+\infty, \quad \bar{A}=\sup _{j \geq 0} \sum_{i=j+1}^{\infty}\left|\tilde{a}_{i, j}\right|<1,
$$

then $\sum_{i=0}^{\infty}\left|x_{i}\right| \leq \bar{B} /(1-\bar{A})<+\infty$, and consequently, $\lim _{i \rightarrow \infty} x_{i}=0$.

Proof. By (2.3),

$$
\begin{aligned}
\sum_{k=0}^{i}\left|x_{k}\right| & \leq \sum_{k=0}^{i}\left|\tilde{b}_{k}\right|+\sum_{k=0}^{i} \sum_{j=0}^{k-1}\left|\tilde{a}_{k, j}\right|\left|x_{j}\right| \\
& \leq\left(\sum_{k=0}^{i}\left|\tilde{b}_{k}\right|\right)+\sum_{j=0}^{i-1}\left(\sum_{k=j+1}^{i}\left|\tilde{a}_{k, j}\right|\right)\left|x_{j}\right| \\
& \leq\left(\sum_{k=0}^{i}\left|\tilde{b}_{k}\right|\right)+\left(\sup _{j \geq 0} \sum_{k=j+1}^{\infty}\left|\tilde{a}_{k, j}\right|\right)\left(\sum_{j=0}^{i-1}\left|x_{j}\right|\right) .
\end{aligned}
$$

Therefore, by (2.19), we have that

$$
\sum_{k=0}^{i}\left|x_{k}\right| \leq \frac{\sum_{k=0}^{i}\left|\tilde{b}_{k}\right|}{\left\{1-\left(\sup _{j \geq 0} \sum_{k=j+1}^{\infty}\left|\tilde{a}_{k, j}\right|\right)\right\}} \leq \frac{\bar{B}}{1-\bar{A}}<+\infty,
$$

and then, $\lim _{i \rightarrow \infty} x_{i}=0$.

In the case (1.1) is linear,

$$
x_{i}=b_{i}-\sum_{j=0}^{i} a_{i, j} x_{j}, \quad i \geq 0,
$$

the following theorem is easily proved. 
Theorem 2.4. For the linear equation (2.22), assume inf $\operatorname{i\geq 0}_{i, i}>-1$, and for

$$
\begin{aligned}
\gamma & =1+\min \left(0, \inf _{i \geq 0} a_{i, i}\right), \\
\tilde{c}_{i, j} & =\frac{a_{i-1, j}-a_{i, j}}{\gamma}, \quad i-2 \geq j \geq 0, \\
\tilde{c}_{i, i-1} & =\frac{1+a_{i-1, i-1}-a_{i, i-1}}{\gamma}, \quad i \geq 1, \\
\tilde{d}_{0} & =\frac{b_{0}}{\gamma}, \quad \tilde{d}_{i}=\frac{b_{i}-b_{i-1}}{\gamma}, \quad i \geq 1 .
\end{aligned}
$$

(i) Suppose that

$$
\sup _{0 \leq j \leq i-1}\left|\tilde{c}_{i, j}\right|=C<+\infty, \quad D=\sup _{i \geq 0}\left|\tilde{d}_{i}\right|<+\infty .
$$

Then, $\left|x_{i}\right| \leq(1+C)^{i} D, i \geq 0$. In particular, if there exists a positive integer $i_{0}$ such that

$$
\bar{C}_{0}=\sup _{i \geq i_{0}} \sum_{j=i_{0}}^{i-1}\left|\widetilde{c}_{i, j}\right|<1,
$$

then $x_{i}$ is bounded and

$$
\left|x_{i}\right| \leq \frac{(1+C)^{i_{0}} D}{1-\bar{C}_{0}}<+\infty, \quad i \geq i_{0}
$$

Moreover, if

$$
\lim _{i \rightarrow \infty}\left(b_{i}-b_{i-1}\right)=0, \quad \lim _{i \rightarrow \infty}\left(\sum_{j=0}^{i_{0}-1}\left|a_{i, j}\right|\right)=0,
$$

then $\lim _{i \rightarrow \infty} x_{i}=0$.

(ii) If

$$
\bar{C}=\sup _{j \geq 0} \sum_{i=j+1}^{\infty}\left|\tilde{c}_{i, j}\right|<1, \quad \bar{D}=\frac{\sum_{i=0}^{\infty}\left|b_{i+1}-b_{i}\right|+\left|b_{0}\right|}{\gamma}<+\infty
$$

then $\sum_{i=0}^{\infty}\left|x_{i}\right| \leq \bar{D} /(1-\bar{C})<+\infty$, and consequently, $\lim _{i \rightarrow \infty} x_{i}=0$.

Proof. By (2.22), we obtain that

$$
\left(1+a_{i, i}\right) x_{i}=\left(b_{i}-b_{i-1}\right)+\left(1+a_{i-1, i-1}-a_{i, i-1}\right) x_{i-1}+\sum_{j=0}^{i-2}\left(a_{i-1, j}-a_{i, j}\right) x_{j}, \quad i \geq 1 .
$$

Then, we have that

$$
\left|x_{i}\right| \leq\left|\tilde{d}_{i}\right|+\sum_{j=0}^{i-1}\left|\tilde{c}_{i, j}\right|\left|x_{j}\right|, \quad i \geq 1
$$

Thus, analogously to the proofs of Theorems 2.2 and 2.3, we obtain the conclusion of this theorem. 


\section{Nonnegative coefficients}

In this section, we focus on the solutions of (1.1) with (1.2) and

$$
a_{i, j} \geq 0, \quad i \geq j \geq 0
$$

Such discrete equations are useful, above all, in the investigations on the behavior of the solution of some numerical methods when used to solve nonlinear heat flow in a material with memory (see [14] and the bibliography therein). Let us start with the following lemma, which describes some aspects of the solution of (1.1)-(1.2) with (3.1) when $x_{i}$ has a sign eventually constant for all $i \geq 0$. The utility of this lemma is not in itself, but as an instrument to prove some of the next theorems (see Theorems 3.4, 4.1 and 4.3).

Lemma 3.1. Let $\left\{x_{i}\right\}_{i=0}^{\infty}$ be the solution of (1.1) and assume that

(i) $\left|b_{i}\right| \leq B$ and $a_{i, j} \leq A_{j}$ for each $i, j \geq 0$;

(ii) there exists $i_{0}>0$ such that $x_{i} \geq 0$ (resp., $x_{i} \leq 0$ ) for any $i \geq i_{0}$,

then

$$
\left|\widetilde{c}_{i}\right| \leq C, \quad 0 \leq x_{i} \leq \tilde{c}_{i} \quad\left(\text { resp., } 0 \geq x_{i} \geq \tilde{c}_{i}\right) \forall i \geq i_{0},
$$

where $\widetilde{c}_{i}=b_{i}-\sum_{j=0}^{i_{0}-1} a_{i, j} f_{i-j}\left(x_{j}\right)$ and $C$ is a positive constant.

Moreover, assume that one of the following conditions holds:

(iii $)_{1} \lim _{i \rightarrow \infty} \widetilde{c}_{i}=0$,

(iiii $) a=\liminf _{j \rightarrow \infty}\left(\liminf _{i \rightarrow \infty} a_{i, j}\right)>0$ and there exists a strictly increasing function $f(x)$ on $(-\infty,+\infty)$ such that $f(0)=0$ and $\inf _{j \geq 0} f_{j}(x) \geq f(x), x \in[0,+\infty)$ (resp., $\inf _{j \geq 0} \bar{f}_{j}(x) \leq$ $\underline{f}(x), x \in(-\infty, 0])$,

then $\lim _{i \rightarrow \infty} x_{i}=0$.

Furthermore, if, in addition to (iii 2 ), there exists a positive constant $\delta$ such that $f(x) \geq \delta x, x \in$ $[0,+\infty)(\operatorname{resp} ., \underline{f}(x) \leq \delta x, x \in(-\infty, 0])$, then $\sum_{i=i_{0}}^{\infty}\left|x_{i}\right| \leq\left(\sup _{i \geq i_{0}}\left|\widetilde{c}_{i}\right|\right) /(\delta a)<+\infty$.

Proof. Since $a_{i, j} \leq A_{j},\left|b_{i}\right| \leq B$ and $f(x)$ is a continuous function, then $\left|\widetilde{c}_{i}\right|$ is bounded. Assume that there exists a nonnegative integer $i_{0}$ such that $x_{i} \geq 0$ for any $i \geq i_{0}$ (the analysis of the case $x_{i} \leq 0$ for all $i \geq i_{0}$ is analogous). Then, by the fact that, for the main hypothesis (1.2), $f_{i-j}(x)>0$ whenever $x>0$, we have

$$
0 \leq x_{i}=\left\{b_{i}-\sum_{j=0}^{i_{0}-1} a_{i, j} f_{i-j}\left(x_{j}\right)\right\}-\sum_{j=i_{0}}^{i} a_{i, j} f_{i-j}\left(x_{j}\right) \leq \tilde{c}_{i}, \quad i \geq i_{0} .
$$

Hence, the first part of the lemma is proved. Consider now the two cases ( $\left.\mathrm{iii}_{1}\right)$ and $\left(\mathrm{iii}_{2}\right)$ separately.

Case (iii 1 ): $\lim _{i \rightarrow \infty} \widetilde{c}_{i}=0$ of course implies $\lim _{i \rightarrow \infty} x_{i}=0$. 
Case (iii 2 ): put $\lim \sup _{i \rightarrow \infty} x_{i}=\bar{x}$. Assume that $\bar{x}>0$, and let $\left\{x_{i_{k}}\right\}_{k=0}^{\infty}$ be a subsequence of $\left\{x_{i}\right\}_{i=i_{0}}^{\infty}$ such that $\lim _{k \rightarrow \infty} x_{i_{k}}=\bar{x}$. Then, one can prove that $\lim _{\sup _{k \rightarrow \infty}} \sum_{j=i_{0}}^{i_{k}} f\left(x_{j}\right)=+\infty$. By (1.1) and assumptions, we have

$$
x_{i_{k}}+\sum_{j=i_{0}}^{i_{k}} a_{i_{k}, j} f\left(x_{j}\right) \leq \widetilde{c}_{i_{k}}
$$

Therefore,

$$
+\infty>\limsup _{k \rightarrow \infty} \widetilde{c}_{i_{k}} \geq \limsup _{k \rightarrow \infty}\left\{x_{i_{k}}+\sum_{j=i_{0}}^{i_{k}} a_{i_{k}, j} \underline{f}\left(x_{j}\right)\right\} \geq \bar{x}+\left\{\liminf _{j \rightarrow \infty}\left(\liminf _{i \rightarrow \infty} a_{i, j}\right)\right\} \limsup _{k \rightarrow \infty} \sum_{j=i_{0}}^{i_{k}} f\left(x_{j}\right)
$$

which is a contradiction because $\liminf _{j \rightarrow \infty}\left(\liminf _{i \rightarrow \infty} a_{i, j}\right)>0$ and $\lim \sup _{k \rightarrow \infty} \sum_{j=i_{0}}^{i_{k}} \underline{f}\left(x_{j}\right)=$ $+\infty$. Hence, we have $\bar{x}=\lim _{i \rightarrow \infty} x_{i}=0$.

In addition, suppose that there exists a positive constant $\delta$ such that $f(x) \geq \delta x, x \in$ $[0,+\infty)$. Then, we have that

$$
x_{i}+\delta \sum_{j=i_{0}}^{i} a_{i, j} x_{j} \leq \widetilde{c}_{i}, \quad i \geq i_{0} .
$$

Thus, from $a=\liminf _{j \rightarrow \infty}\left(\liminf _{i \rightarrow \infty} a_{i, j}\right)>0$, we conclude that $0 \leq \sum_{i=i_{0}}^{\infty} x_{i} \leq\left(\sup _{i \geq i_{0}} \widetilde{c}_{i}\right) /(\delta a)<$ $+\infty$.

The proof is completely analogous when there exists a nonnegative integer $i_{0}$ such that $x_{i} \leq 0$ for any $i \geq i_{0}$.

Remark 3.2. Observe that in the linear case (2.22), the last conditions of Lemma 3.1 are satisfied whenever $\delta=1$ and $f(x)=x$.

Hereafter, we investigate on the boundedness of the solution of (1.1)-(1.2) when

$$
f(x) \neq x, \quad \lim _{x \rightarrow-\infty} f(x)>-\infty .
$$

Lemma 3.3. Let $\left\{x_{i}\right\}_{i=0}^{\infty}$ be the solution of (1.1) with (1.2) and (3.7), and assume that

$$
\sup _{i \geq 0}\left|b_{i}\right|<+\infty, \quad \lambda=\sup _{i \geq 0} \sum_{j=0}^{i} a_{i, j}<+\infty,
$$

then $\left|x_{i}\right|$ is bounded.

Proof. Let $\bar{b}$ be the bound for $\left|b_{i}\right|, i \geq 0$ and $\lim _{x \rightarrow-\infty} f(x)=-\beta>-\infty$. Let us write $\sum_{j=0}^{i} a_{i, j} f_{i-j}\left(x_{j}\right)$ as the sum of the following two contributions:

$$
\begin{aligned}
x_{i} & =b_{i}-\sum_{j=0}^{i} a_{i, j} f_{i-j}\left(x_{j}\right) \\
& =b_{i}-\sum_{l=1}^{i_{p}} a_{i, j_{l}} f_{i-j_{l}}\left(x_{j_{l}}\right)-\sum_{l=1}^{i_{n}} a_{i, k_{l}} f_{i-k_{l}}\left(x_{k_{l}}\right),
\end{aligned}
$$


where $f_{i-j_{l}}\left(x_{j_{l}}\right) \geq 0, l=1, \ldots, i_{p}, f_{i-k_{l}}\left(x_{k_{l}}\right)<0, l=1, \ldots, i_{n}$, and $i_{p}+i_{n}=i+1$. Therefore, since (1.2), (3.1), (3.7), and (3.8) hold, we have that

$$
\begin{aligned}
& x_{i} \leq \bar{b}-\sum_{l=1}^{i_{n}} a_{i, k_{l}} f_{i-k_{l}}\left(x_{k_{l}}\right) \leq \bar{b}-\sum_{l=1}^{i_{n}} a_{i, k_{l}} f\left(x_{k_{l}}\right) \leq \bar{b}+\lambda \beta \\
& x_{i} \geq-\bar{b}-\sum_{l=1}^{i_{p}} a_{i, j_{l}} f_{i-j_{l}}\left(x_{j_{l}}\right) \geq-\bar{b}-f(\bar{b}+\beta \lambda) \sum_{l=1}^{i_{p}} a_{i, j_{l}} \geq-\bar{b}-\lambda f(\bar{b}+\beta \lambda) .
\end{aligned}
$$

Thus, $x_{i}$ is bounded and the proof is complete.

As an example we consider the equation

$$
x_{i}=1-\sum_{j=0}^{i} \frac{1}{(i+1) 2^{i-j}} \frac{e^{x_{j}}-1}{1+x_{j}^{2}},
$$

in this case $f(x)=e^{x}-1, \bar{b}=\sup _{i \geq 0}\left|b_{i}\right|=1, \lambda=\sup _{i \geq 0} \sum_{j=0}^{i} 1 /\left((i+1) 2^{i-j}\right)=1$, and $\beta=$ $\lim _{x \rightarrow-\infty}\left(e^{x}-1\right)=-1$. Hence,

$$
-\frac{1}{e} \leq x_{i} \leq 0
$$

Another example is given by the explicit equation

$$
x_{i}=(-1)^{i}-\frac{1}{10} \sum_{j=0}^{i-1} \frac{1}{(i+1) 2^{i-j}} \frac{e^{x_{j}}-1}{1+x_{j}^{2}} .
$$

Here $\bar{b}=1$ and $\lambda=1 / 40$, hence

$$
-1-\frac{1}{40}\left(e^{-1 / 40}-1\right) \leq x_{i} \leq \frac{39}{40} .
$$

From Figure 1 it is clear that the bounds established by Lemma 3.3 (represented by dotted lines) may be quite sharp. We are able to prove the following result.

Theorem 3.4. Assume that $f(x) \neq x$ is continuous on $(-\infty,+\infty)$,

$$
\begin{gathered}
\bar{r}=\limsup _{i \rightarrow \infty} \sum_{j=0}^{i-1} a_{i, j}<+\infty, \quad-\bar{r} f(-\bar{r} f(L))>L, \quad \text { for any } L<0, \\
\lim _{i \rightarrow \infty} b_{i}=0, \quad \lim _{i \rightarrow \infty} a_{i, j}=0,
\end{gathered}
$$

for all $j \geq 0$. Then $\lim _{i \rightarrow \infty} x_{i}=0$.

Proof. Let $\underline{x}=\liminf _{i \rightarrow \infty} x_{i}$ and assume $\underline{x}<0$. Since we are in the hypotheses of Lemma 3.3, $\left|x_{i}\right|$ is bounded and then $M=\sup _{i \geq 0}\left|f\left(x_{i}\right)\right|<+\infty$. For any fixed $\epsilon>0$, consider a continuous 


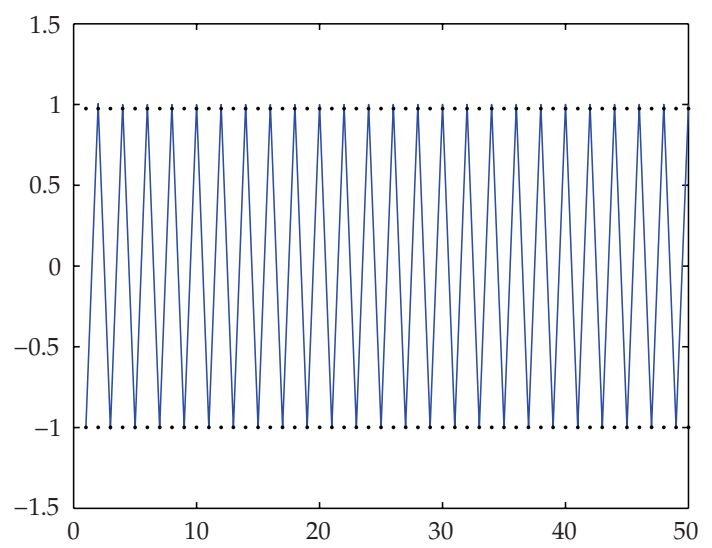

Figure 1: Plot of (3.13) and its bounds given by (3.14).

function $F(x)=-(\bar{r}+x) f(-(\bar{r}+x) f(\underline{x}-x)+x)-2 x$ on $[0, \epsilon]$. Then, by $F(0)=-\bar{r} f(-\bar{r} f(\underline{x}))>\underline{x}$, there exists a constant $0<\epsilon_{0}<\epsilon$ such that

$$
F\left(\epsilon_{0}\right)=-\left(\bar{r}+\epsilon_{0}\right) f\left(-\left(\bar{r}+\epsilon_{0}\right) f\left(\underline{x}-\epsilon_{0}\right)+\epsilon_{0}\right)-2 \epsilon_{0}>\underline{x} .
$$

For the above $\epsilon_{0}>0$, there exists a positive integer $i_{0}$ such that $x_{i}>\underline{x}-\epsilon_{0}$ and $\sum_{j=0}^{i-1} a_{i, j}<\bar{r}+\epsilon_{0}$, for any $i \geq i_{0}$. By Assumption (3.16), we have that for $\epsilon_{1}=\epsilon_{0} /(1+M)>0$, there exists a positive integer $i_{1} \geq i_{0}$ such that

$$
\left|b_{i}\right|<\epsilon_{1}, \quad \sum_{j=0}^{i_{1}-1} a_{i, j}<\epsilon_{1} \quad \text { for any } i \geq i_{1} .
$$

Then, for $i \geq i_{1}$ and $\tilde{c}_{i}=b_{i}-\sum_{j=0}^{i_{1}-1} a_{i, j} f_{i-j}\left(x_{j}\right)$, we have that

$$
\left|\widetilde{c}_{i}\right| \leq\left|b_{i}\right|+\left(\sum_{j=0}^{i_{1}-1} a_{i, j}\right) M<\epsilon_{1}+\epsilon_{1} M=\epsilon_{0} .
$$

Let us rewrite (1.1) in the following form:

$$
x_{i}=\tilde{c}_{i}-\sum_{l=1}^{i_{p}} a_{i, j_{l}} f_{i-j_{l}}\left(x_{j_{l}}\right)-\sum_{l=1}^{i_{n}} a_{i, k_{l}} f_{i-k_{l}}\left(x_{k_{l}}\right),
$$

where $f_{i-j_{l}}\left(x_{j_{l}}\right) \geq 0$, for $l=1, \ldots, i_{p}$ and $f_{i-k_{l}}\left(x_{k_{l}}\right)<0$, for $l=1, \ldots, i_{n}$ and $i_{p}+i_{n}=i-i_{1}+1$. Thus,

$$
x_{i} \leq \widetilde{c}_{i}-\sum_{l=0}^{i_{n}} a_{i, k_{l}} f\left(x_{k_{l}}\right)<\epsilon_{0}-\left(\bar{r}+\epsilon_{0}\right) f\left(\underline{x}-\epsilon_{0}\right), \quad \forall i \geq i_{1}
$$

and, since $f(x)$ is an increasing function, we have that, for all $j_{l} \geq i_{1}$,

$$
f\left(x_{j_{l}}\right)<f\left(\epsilon_{0}-\left(\bar{r}+\epsilon_{0}\right) f\left(\underline{x}-\epsilon_{0}\right)\right) .
$$


Since we are in the hypothesis that the coefficients $a_{i, j}$ are nonnegative, it follows that

$$
\begin{aligned}
-\sum_{l=0}^{i_{p}} a_{i, j_{l}} f\left(x_{j_{l}}\right) & >-\sum_{l=0}^{i_{p}} a_{i, j_{l}} f\left(\epsilon_{0}-\left(\bar{r}+\epsilon_{0}\right) f\left(\underline{x}-\epsilon_{0}\right)\right) \\
& >-\left(\bar{r}+\epsilon_{0}\right) f\left(\epsilon_{0}-\left(\bar{r}+\epsilon_{0}\right) f(\underline{x}-\epsilon)\right) .
\end{aligned}
$$

In conclusion, from

$$
-\sum_{l=0}^{i_{p}} a_{i, j_{l}} f\left(x_{j_{l}}\right)>-\left(\bar{r}+\epsilon_{0}\right) f\left(\epsilon_{0}-\left(\bar{r}+\epsilon_{0}\right) f\left(\underline{x}-\epsilon_{0}\right)\right)=F\left(\epsilon_{0}\right)+2 \epsilon_{0}
$$

and by using (3.20), (3.19), and (3.17), the following inequality holds:

$$
x_{i} \geq \widetilde{c}_{i}-\sum_{l=0}^{i_{p}} a_{i, j_{l}} f\left(x_{j_{l}}\right)>-\epsilon_{0}-\left(\bar{r}+\epsilon_{0}\right) f\left(\epsilon_{0}-\left(\bar{r}+\epsilon_{0}\right) f(\underline{x}-\epsilon)\right)=F\left(\epsilon_{0}\right)+\epsilon_{0}>\underline{x}+\epsilon_{0} .
$$

This result contradicts the lim inf definition. Hence, $\underline{x} \geq 0$, so $x_{i}$ are eventually nonnegative. Since it is easy to see that we are in the hypotheses of Lemma 3.1 (case (iii 1$)$ ), then $\lim _{i \rightarrow \infty} x_{i}$ $=0$.

Remark 3.5. Once again, in the convolution case, the first part of (3.15) implies the second one of (3.16).

For the special case $f(x)=\left(e^{\alpha x}-1\right) / \alpha, \alpha>0$, we establish the following sufficient condition from Theorem 3.4.

Theorem 3.6. Suppose that $f(x)=\left(e^{\alpha x}-1\right) / \alpha, \alpha>0$ and assume that

$$
\text { (3.16), the first part of (3.15) with } \bar{r} \leq 1
$$

hold, then the solution $x_{i}$ of (1.1) tends to zero as $i$ tends to infinity.

Proof. Put $\varphi(x)=-\bar{r} f(x),-\infty<x<+\infty$. Since $\varphi^{\prime}(x)=-\bar{r} e^{\alpha x}<0$ for $x \in(-\infty,+\infty)$, hence $\varphi(x)$ is a strictly monotone decreasing function in $(-\infty,+\infty)$. Now, we will prove that $\varphi(\varphi(x))>x$, for $-\infty<x<0$.

Let $g(x)=\varphi(\varphi(x))-x$ for $-\infty<x<0$. Then we have that

$$
\begin{aligned}
g(x) & =\frac{\bar{r}\left\{1-\exp \left(-\bar{r}\left(e^{\alpha x}-1\right)\right)\right\}}{\alpha}-x, \\
g^{\prime}(x) & =\bar{r}\left\{\bar{r} e^{\alpha x} \exp \left(\bar{r}-\bar{r} e^{\alpha x}\right)\right\}-1 .
\end{aligned}
$$

By recalling that $\bar{r}<1$ and $x<0$, we have $0 \leq \bar{r} e^{\alpha x}<1$. Since the function $y e^{-y}$ is increasing for $0 \leq y \leq 1$, there results $\left(\bar{r} e^{\alpha x}\right) e^{-\bar{r} e^{\alpha x}} \leq e^{-1}$. Thus, $g^{\prime}(x) \leq \bar{r} \exp (\bar{r}-1)-1 \leq \bar{r}-1 \leq 0$. Hence, we have that $\varphi(\varphi(x))>x$, for $-\infty<x<0$. Thus, for $f(x)=\left(e^{\alpha x}-1\right) / \alpha$ with $\alpha>0$, the second of (3.15) is true and, by Theorem 3.4, we have $\lim _{i \rightarrow \infty} x_{i}=0$.

Remark 3.7. From the proof of Theorem 3.6 it is clear that the second part of (3.15) is satisfied by $f(x)=\left(e^{\alpha x}-1\right) / \alpha(\alpha>0)$. By playing with $\alpha$, this allows us to consider a wide variety of functions $f_{i}$ which satisfy (1.2). For instance, in the cases $\alpha=1 / 2$ and $\alpha=1$ the stained areas in Figure 2 represent the admissible regions for the functions $f_{i}, i=0,1, \ldots$, respectively (the solid lines show, as an example, the graphs of $f_{i}(x)=x /\left(1+x^{2}\right)$ and $\left.f_{i}(x)=\left(e^{x}-1\right) /\left(1+x^{2}\right)\right)$. 


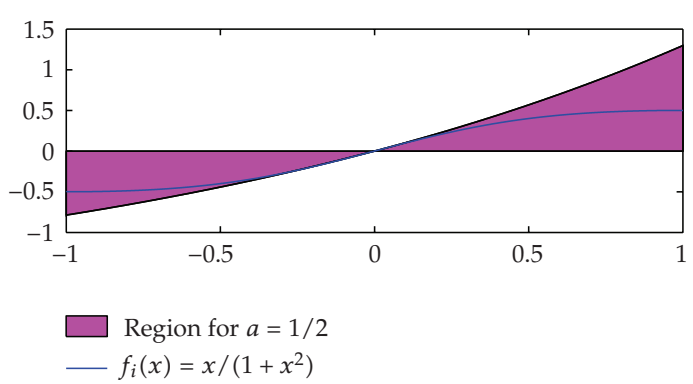

(a)

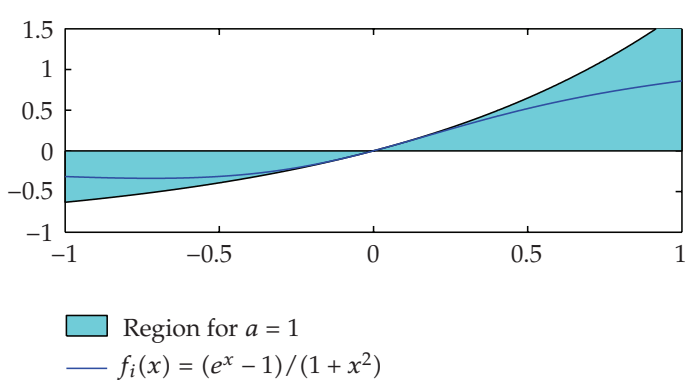

(b)

Figure 2: Plots of some admissible regions for $f_{i}$ according to Theorem 3.6.

\section{Monotonic nonnegative coefficients}

In this section, for (1.1), first, we consider the case that

$$
0 \leq a_{i, j} \leq a_{i-1, j}, \quad 0 \leq j \leq i-1, \quad\left|f_{i-1}(x)\right| \geq\left|f_{i}(x)\right|, \quad i \geq 1 .
$$

We provide the following theorem which generalizes [15, Theorem 2.1] to the nonlinear case.

Theorem 4.1. In addition to condition (4.1), suppose that

$$
b_{i} \geq b_{i-1} \geq 0 \quad\left(\text { resp., } b_{i} \leq b_{i-1} \leq 0\right), \quad i \geq 1 .
$$

Then, any solution $x_{i}$ of (1.1) satisfies $0 \leq x_{i} \leq b_{i}$ (resp., $0 \geq x_{i} \geq b_{i}$ ), $i \geq 0$. Moreover, if $\left|b_{i}\right| \leq B$, for all $i \geq 0, a=\liminf _{j \rightarrow \infty}\left(\lim _{i \rightarrow \infty} a_{i, j}\right)>0$ and there exists a strictly increasing function $f(x)$ on $(-\infty,+\infty)$ such that $\underline{f}(0)=0$ and $\inf _{j \geq 0} f_{j}(x) \geq \underline{f}(x), x \in[0,+\infty)\left(\right.$ resp., $\inf _{j \geq 0} f_{j}(x) \leq \underline{f}(\bar{x}), x \in$ $(-\infty, 0])$, then $\lim _{i \rightarrow \infty} x_{i}=0$.

In addition, if there exists a positive constant $\delta$ such that $f(x) \geq \delta x, x \in[0,+\infty)$ (resp., $\underline{f}(x) \leq \delta x, x \in(-\infty, 0])$, then $\sum_{i=0}^{\infty}\left|x_{i}\right| \leq\left(\sup _{i \geq 0}\left|b_{i}\right|\right) /(\delta a)<+\infty$.

Proof. We prove the theorem in the case $b_{i} \geq b_{i-1} \geq 0, i \geq 1$, the proof for $b_{i} \leq b_{i-1} \leq 0, i \geq 1$ is perfectly symmetric. Then by (1.1), we have that

$$
x_{0}+a_{0,0} f_{0}\left(x_{0}\right)=b_{0} \geq 0,
$$

hence, for the properties of $f_{i}(x)$ described in (1.2), it has to be $x_{0} \geq 0$.

Proceeding by induction, suppose that $x_{j} \geq 0,0 \leq j \leq i-1, i \geq 1$. By (1.1),

$$
\begin{aligned}
& x_{i}+a_{i, i} f_{0}\left(x_{i}\right)=b_{i}-\sum_{j=0}^{i-1} a_{i, j} f_{i-j}\left(x_{j}\right), \\
& 0=x_{i-1}-b_{i-1}+\sum_{j=0}^{i-1} a_{i-1, j} f_{i-j-1}\left(x_{j}\right),
\end{aligned}
$$


and hence, by adding the two relations and taking into account that, for the second part of (4.1), $f_{i-j-1}(x) \geq f_{i-j}(x)$, we have that

$$
\begin{aligned}
x_{i}+a_{i, i} f_{0}\left(x_{i}\right) & \geq\left(b_{i}-b_{i-1}\right)+x_{i-1}+\sum_{j=0}^{i-2}\left(a_{i-1, j}-a_{i, j}\right) f_{i-j}\left(x_{j}\right) \\
& \geq b_{i}-b_{i-1} \geq 0, \quad i \geq 1 .
\end{aligned}
$$

So we have that $x_{i} \geq 0, i \geq 0$ and, from (1.1), $x_{i} \leq b_{i}, i \geq 0$.

Thus, we are in the hypotheses of Lemma 3.1 part ( $\left.\mathrm{iii}_{2}\right)$ and, hence, we get the thesis.

Observe that when (1.1) is linear, the last condition of Theorem 4.1 is satisfied by choosing $\delta=1$ and $f(x)=x$. In this case, the hypotheses of Theorem 4.1 include, as particular cases, those of [15, Theorem 2.1]. In particular we note that, as Theorem 4.1 prove the summability of the solution $x_{i}$, it is interesting when applied to the equation satisfied by the fundamental matrix of a Volterra difference equation (see, e.g., [15, equation (1.4)]). Namely, in [15] it is underlined that such a result can be employed in the study of the stability of some numerical methods.

A simple application of Theorem 4.1 in the linear case is given by the following example.

Example 4.2. Let us consider the difference equation

$$
x_{i}=b-\sum_{j=0}^{i} c x_{j}, \quad i \geq 0,
$$

whose solution is given by

$$
x_{i}=\frac{b}{(1+c)^{i+1}}, \quad i \geq 0
$$

Then, for $a>0$ and $b \geq 0$, all the conditions in Theorem 4.1 are satisfied with $\delta=1$, which implies $\lim _{i \rightarrow \infty} x_{i}=0$ and $\sum_{i=0}^{+\infty} x_{i} \leq(b / a)<+\infty$. Observe that in this case the bound coincides with the exact value of the sum of the series.

Next, we provide the following theorem whose proof is a direct extension of the proof of Crisci et al. [6, Theorem 2.1], which gives a priori bound for the solution $x_{i}$ of (1.1) depending on the forcing terms $b_{i}$.

Theorem 4.3. In addition to the conditions (4.1), assume that

$$
\sup _{i \geq 1}^{i-1} \sum_{j=0}\left|b_{j+1}-b_{j}\right|<+\infty .
$$

Then, any solution $x_{i}$ of (1.1) is bounded and satisfies

$$
\left|x_{i}\right| \leq \sum_{j=0}^{i-1}\left|b_{j+1}-b_{j}\right|+\left|b_{0}\right|, \quad i \geq 0 .
$$


Moreover, suppose that $a=\liminf _{j \rightarrow \infty}\left(\lim _{i \rightarrow \infty} a_{i, j}\right)>0$ and there exists a strictly increasing function $f(x)$ on $(-\infty,+\infty)$ such that $f(0)=0$ and $\inf _{j \geq 0} f_{j}(x) \geq f(x), x \in[0,+\infty)\left(\right.$ resp., $\inf _{j \geq 0} f_{j}(x) \leq$ $\underline{f}(x), x \in(-\infty, 0])$, then $\lim _{i \rightarrow \infty} x_{i}=0$.

In addition, if there exists a positive constant $\delta$ such that $f(x) \geq \delta x, x \in[0,+\infty)$ (resp., $\underline{f}(x) \leq \delta x, x \in(-\infty, 0])$, then $\sum_{i=0}^{\infty}\left|x_{i}\right| \leq(2 / \delta a)\left(\sum_{j=0}^{\infty}\left|b_{j+1}-b_{j}\right|-\left|b_{0}\right|\right)<+\infty$.

Proof. Consider the two possible subcases: (a) $x_{0} \geq 0$, and (b) $x_{0}<0$.

(a) Assume $x_{0} \geq 0$.

If $x_{j} \geq 0,0 \leq j \leq i$, then by (1.1), we get $b_{i} \geq 0$ and $0 \leq x_{i} \leq b_{i}$. Hence, (4.9) holds if $\left\{x_{j}\right\}_{j=0}^{i}$ is oscillatory about 0 . Let $m_{0}=-1$ and denote by $l_{1}$ the time moment of the first passage of the solution $x_{i}$ through the zero, that is,

$$
x_{j} \geq 0 \quad \text { for } m_{0}+1=0 \leq j \leq l_{1}, \quad x_{l_{1}+1}<0 .
$$

The time moment of the following passage through the zero of the solution after $l_{1}$ is denoted by $m_{1}$, that is,

$$
x_{j} \leq 0 \quad \text { for } l_{1}+1 \leq j \leq m_{1}, \quad x_{m_{1}+1}>0 .
$$

In a similar way, we introduce the indexes $l_{p}, m_{p}, p \geq 1$ as follows:

$$
\begin{aligned}
& x_{j} \geq 0 \quad \text { for } m_{p-1}+1 \leq j \leq l_{p}, \quad x_{l_{p}+1}<0, \\
& x_{j} \leq 0 \quad \text { for } l_{p}+1 \leq j \leq m_{p}, \quad x_{m_{p}+1}>0 .
\end{aligned}
$$

(1) Consider that $m_{k}+1 \leq i \leq l_{k+1}$, hence $x_{i} \geq 0$. Then, from (1.1), we have that

$$
\begin{aligned}
& x_{i}=b_{i}-\sum_{j=m_{k}+1}^{i} a_{i, j} f_{i-j}\left(x_{j}\right)-\sum_{j=0}^{m_{k}} a_{i, j} f_{i-j}\left(x_{j}\right), \\
& 0=x_{m_{k}}-b_{m_{k}}+\sum_{j=l_{k}+1}^{m_{k}} a_{m_{k}, j} f_{m_{k}-j}\left(x_{j}\right)+\sum_{j=0}^{l_{k}} a_{m_{k}, j} f_{m_{k}-j}\left(x_{j}\right), \\
& 0=-x_{l_{k}}+b_{l_{k}}-\sum_{j=m_{k-1}+1}^{l_{k}} a_{l_{k}, j} f_{l_{k}-j}\left(x_{j}\right)-\sum_{j=0}^{m_{k-1}} a_{l_{k}, j} f_{l_{k}-j}\left(x_{j}\right), \\
& 0=x_{m_{k-1}}-b_{m_{k-1}}+\sum_{j=l_{k-1}+1}^{m_{k-1}} a_{m_{k-1}, j} f_{m_{k-1}-j}\left(x_{j}\right)+\sum_{j=0}^{l_{k-1}} a_{m_{k-1}, j} f_{m_{k-1}-j}\left(x_{j}\right), \\
& 0=x_{m_{1}}-b_{m_{1}}+\sum_{j=l_{1}+1}^{m_{1}} a_{m_{1, j}} f_{m_{1}-j}\left(x_{j}\right)+\sum_{j=0}^{l_{1}} a_{m_{1, j}} f_{m_{1}-j}\left(x_{j}\right), \\
& 0=-x_{l_{1}}+b_{l_{1}}-\sum_{j=0}^{l_{1}} a_{l_{1, j}, j} f_{l_{1}-j}\left(x_{j}\right),
\end{aligned}
$$


where every summation of the type $\sum_{j=m_{r-1}+1}^{l_{r}}$ involves only positive $x_{j}$, while $\sum_{j=l_{r}+1}^{m_{r}}$ the negative ones. Now observe that, for $l_{k}+1 \leq j \leq m_{k}$, by using (4.1) and the fact that $m_{k}<i$, we have $\left|f_{m_{k}-j}\left(x_{j}\right)\right| \geq\left|f_{i-j}\left(x_{j}\right)\right|$, furthermore $x_{j} \leq 0$, because of (4.12), hence both $f_{i-j}\left(x_{j}\right)$ and $f_{m_{k}-j}\left(x_{j}\right)$ are less than or equal to zero, thus $f_{m_{k}-j}\left(x_{j}\right) \leq f_{i-j}\left(x_{j}\right)$. By using these considerations it is easy to see that the following inequality holds:

$$
\sum_{j=l_{k}+1}^{m_{k}}\left[a_{m_{k}, j} f_{m_{k}-j}\left(x_{j}\right)-a_{i, j} f_{i-j}\left(x_{j}\right)\right] \leq \sum_{j=l_{k}+1}^{m_{k}}\left(a_{m_{k}, j}-a_{i, j}\right) f_{i-j}\left(x_{j}\right) .
$$

With analogues considerations we get

$$
\begin{gathered}
-\sum_{j=m_{k-1}+1}^{l_{k}}\left[a_{l_{k}, j} f_{l_{k}-j}\left(x_{j}\right)-a_{m_{k}, j} f_{m_{k}-j}\left(x_{j}\right)+a_{i, j} f_{i-j}\left(x_{j}\right)\right] \\
\leq-\sum_{j=m_{k-1}+1}^{l_{k}}\left(a_{l_{k}, j}-a_{m_{k}, j}+a_{i, j}\right) f_{i-j}\left(x_{j}\right), \\
\cdots \\
-\sum_{j=0}^{l_{1}}\left[a_{l_{1}, j} f_{l_{1}-j}\left(x_{j}\right)-a_{m_{1}, j} f_{m_{1}-j}\left(x_{j}\right)+\cdots+a_{i, j} f_{i-j}\left(x_{j}\right)\right] \\
\leq-\sum_{j=0}^{l_{1}}\left(a_{l_{1, j}}-a_{m_{1}, j}+\cdots+a_{i, j}\right) f_{i-j}\left(x_{j}\right) .
\end{gathered}
$$

By adding each side of (4.13) and taking into account (4.14), (4.15), it comes out that

$$
\begin{aligned}
0 \leq x_{i} \leq & \left\{b_{i}-b_{m_{k}}+b_{l_{k}}-b_{m_{k-1}}+\cdots-b_{m_{1}}+b_{l_{1}}\right\}+\left\{x_{m_{k}}-x_{l_{k}}+x_{m_{k-1}}-\cdots+x_{m_{1}}-x_{l_{1}}\right\} \\
& -\sum_{j=m_{k}+1}^{i} a_{i, j} f_{i-j}\left(x_{j}\right)+\sum_{j=l_{k}+1}^{m_{k}}\left(a_{m_{k}, j}-a_{i, j}\right) f_{i-j}\left(x_{j}\right) \\
& -\sum_{j=m_{k-1}+1}^{l_{k}}\left(a_{l_{k}, j}-a_{m_{k}, j}+a_{i, j}\right) f_{i-j}\left(x_{j}\right) \\
& +\sum_{j=l_{k-1}+1}^{m_{k-1}}\left(a_{m_{k-1}, j}-a_{l_{k}, j}+a_{m_{k}, j}-a_{i, j}\right) f_{i-j}\left(x_{j}\right) \\
& \quad \ldots \\
& +\sum_{j=l_{1}+1}^{m_{1}}\left(a_{m_{1}, j}-a_{l_{2}, j}+a_{m_{2}, j}-a_{l_{3}, j}+\cdots+a_{m_{k}, j}-a_{i, j}\right) f_{i-j}\left(x_{j}\right) \\
& -\sum_{j=0}^{l_{1}}\left(a_{l_{1, j}}-a_{m_{1}, j}+a_{l_{2, j}}-a_{m_{2}, j}+\cdots-a_{m_{k}, j}+a_{i, j}\right) f_{i-j}\left(x_{j}\right) .
\end{aligned}
$$


By using the monotonicity of $a_{i, j}$ stated by (4.1) and the main hypothesis (1.2), taking into account (4.12), we have that

$$
0 \leq x_{i} \leq b_{i}-b_{m_{k}}+b_{l_{k}}-b_{m_{k-1}}+\cdots-b_{m_{1}}+b_{l_{1}} \leq \sum_{j=0}^{i-1}\left|b_{j}-b_{j-1}\right|+\left|b_{0}\right| .
$$

(2) Consider that $l_{k}+1 \leq i \leq m_{k}$, hence $x_{i} \leq 0$. Proceeding as above, we have

$$
0 \geq x_{i} \geq-\sum_{j=0}^{i-1}\left|b_{j}-b_{j-1}\right|+\left|b_{0}\right| .
$$

Hence, from (1) and (2), we obtain (4.9). Part (b) of the proof is essentially mirror-like of part (a) and leads once again to (4.9). Thus, any solution $x_{i}$ of (1.1) is bounded and satisfies (4.9).

Moreover, suppose that $a=\liminf _{j \rightarrow \infty}\left(\lim _{i \rightarrow \infty} a_{i, j}\right)>0$ and there exists a strictly increasing function $\underline{f}(x)$ on $(-\infty,+\infty)$ such that $\underline{f}(0)=0$ and $\inf _{j \geq 0} f_{j}(x) \geq \underline{f}(x), x \in[0,+\infty)$ (resp., $\left.\inf _{j \geq 0} f_{j}(x) \leq \bar{f}(x), x \in(-\infty, 0]\right)$.

If there exists a nonnegative integer $i_{0}$ such that $x_{i} \geq 0$ (resp., $x_{i} \leq 0$ ) for any $i \geq i_{0}$, since $\left|b_{i}\right| \leq \sum_{j=0}^{i-1}\left|b_{j}-b_{j-1}\right|+\left|b_{0}\right|$ and $0 \leq a_{i, j} \leq a_{0, j}$, for all $j$, we are in the hypotheses of Lemma 3.1 part $\left(\mathrm{iii}_{2}\right)$ and we obtain $\lim _{i \rightarrow \infty} x_{i}=0$. On the contrary, if such an index does not exist, let $x_{0} \geq 0$ and consider the extract subsequence $\left\{x_{i_{p}}\right\}_{p=0}^{\infty}$ of all the positive values in $\left\{x_{i}\right\}_{i=0}^{\infty}$. Assume that $\bar{x}=\lim \sup _{p \rightarrow+\infty} x_{i_{p}}>0$, then

$$
\limsup _{p \rightarrow+\infty} \sum_{j=0}^{p} \underline{f}\left(x_{i_{j}}\right)=+\infty
$$

Taking into account (4.12), there exists an index $k \geq 0$ such that $m_{k}+1 \leq i_{p} \leq l_{k+1}$, then $x_{i_{p}}$ plays the role of $x_{i}$ in (4.13) and, analogously to (4.16), we have

$$
\begin{aligned}
& x_{i_{p}}+\sum_{j=m_{k}+1}^{i_{p}} a_{i_{p}, j} f_{i_{p}-j}\left(x_{j}\right)+\sum_{j=m_{k-1}+1}^{l_{k}}\left(a_{l_{k}, j}-a_{m_{k}, j}+a_{i_{p}, j}\right) f_{i_{p}-j}\left(x_{j}\right)+\cdots \\
& \quad+\sum_{j=0}^{l_{1}}\left(a_{l_{1}, j}-a_{m_{1}, j}+a_{l_{2}, j}-a_{m_{2}, j}+\cdots-a_{m_{k}, j}+a_{i_{p}, j}\right) f_{i_{p}-j}\left(x_{j}\right) \\
& \leq\left\{b_{i_{p}}-b_{m_{k}}+b_{l_{k}}-b_{m_{k-1}}+\cdots-b_{m_{1}}+b_{l_{1}}\right\} \\
&+\left\{x_{m_{k}}-x_{l_{k}}+x_{m_{k-1}}-\cdots+x_{m_{1}}-x_{l_{1}}\right\}+\sum_{j=l_{k}+1}^{m_{k}}\left(a_{m_{k}, j}-a_{i_{p}, j}\right) f_{i_{p}-j}\left(x_{j}\right) \\
&+\sum_{j=l_{k-1}+1}^{m_{k-1}}\left(a_{m_{k-1}, j}-a_{l_{k}, j}+a_{m_{k}, j}-a_{i_{p}, j}\right) f_{i_{p}-j}\left(x_{j}\right) \\
&+\sum_{j=l_{1}+1}^{m_{1}}\left(a_{m_{1}, j}-a_{l_{2}, j}+a_{m_{2}, j}-a_{l_{3}, j}+\cdots+a_{m_{k}, j}-a_{i_{p}, j}\right) f_{i_{p}-j}\left(x_{j}\right) \\
& \leq b_{i_{p}}-b_{m_{k}}+b_{l_{k}}-b_{m_{k-1}}+\cdots-b_{m_{1}}+b_{l_{1}} \leq \sum_{j=0}^{i_{p}-1}\left|b_{j+1}-b_{j}\right|+\left|b_{0}\right|<+\infty .
\end{aligned}
$$


Hence, since $\underline{f}(x) \leq f_{j}(x)$, for all $x \geq 0$, we have that

$$
\begin{aligned}
& x_{i_{p}}+\sum_{j=m_{k}+1}^{i_{p}} a_{i_{p}, j} \underline{f}\left(x_{j}\right)+\sum_{j=m_{k-1}+1}^{l_{k}}\left(a_{l_{k}, j}-a_{m_{k}, j}+a_{i_{p}, j}\right) \underline{f}\left(x_{j}\right)+\cdots \\
& \quad+\sum_{j=0}^{l_{1}}\left(a_{l_{1}, j}-a_{m_{1}, j}+a_{l_{2}, j}-a_{m_{2}, j}+\cdots-a_{m_{k}, j}+a_{i_{p}, j}\right) \underline{f}\left(x_{j}\right) \leq \sum_{j=0}^{i_{p}-1}\left|b_{j+1}-b_{j}\right|+\left|b_{0}\right|<+\infty,
\end{aligned}
$$

and so, since only positive quantities are involved, we get

$$
a\left(\sum_{j=m_{k}+1}^{i_{p}} \underline{f}\left(x_{j}\right)+\sum_{j=m_{k-1}+1}^{l_{k}} \underline{f}\left(x_{j}\right)+\cdots+\sum_{j=0}^{l_{1}} \underline{f}\left(x_{j}\right)\right) \leq \sum_{j=0}^{i_{p}-1}\left|b_{j+1}-b_{j}\right|+\left|b_{0}\right|<+\infty .
$$

Passing to the $\lim \sup$ as $p \rightarrow+\infty$, we have that

$$
\underset{p \rightarrow+\infty}{\operatorname{alimsup}}\left(\sum_{j=m_{k}+1}^{i_{p}} f\left(x_{j}\right)+\sum_{j=m_{k-1}+1}^{l_{k}} f\left(x_{j}\right)+\cdots+\sum_{j=0}^{l_{1}} \underline{f}\left(x_{j}\right)\right)<+\infty .
$$

Taking into account that all the $x_{j}$ involved in the summations above form the extract $\left\{x_{i_{p}}\right\}_{p=0}^{+\infty}$ of the positive values in $\left\{x_{i}\right\}_{i=0}^{+\infty}$, we get $\lim _{\sup _{p \rightarrow+\infty}} \sum_{j=0}^{p} f\left(x_{i_{j}}\right)<+\infty$, which is a contradiction with (4.19), so $\bar{x}=0$. An analogous proof on the extract subsequence of all negative values of $\left\{x_{i}\right\}_{i=0}^{+\infty}$ leads to $\lim \inf _{n \rightarrow+\infty} x_{i_{n}}=0$. The same happens when $x_{0}<0$. Hence, in conclusion, we obtain that $\lim _{i \rightarrow \infty} x_{i}=0$.

In addition, suppose that there exists a positive constant $\delta$ such that $f(x) \geq \delta x, x \in$ $[0,+\infty)$. Then, by $(4.22)$ and the fact that $a$ is strictly positive, we conclude that $0 \leq$ $\sum_{i=0}^{\infty} \max \left(0, x_{i}\right) \leq 1 / \delta a\left(\sum_{j=0}^{\infty}\left|b_{j+1}-b_{j}\right|+\left|b_{0}\right|\right)$. Similarly, we obtain that $0 \geq \sum_{i=0}^{\infty} \min \left(0, x_{i}\right) \geq$ $-1 / \delta a\left(\sum_{j=0}^{\infty}\left|b_{j+1}-b_{j}\right|+\left|b_{0}\right|\right)$. Hence, $\sum_{i=0}^{\infty}\left|x_{i}\right| \leq 2 / \delta a\left(\sum_{j=0}^{\infty}\left|b_{j+1}-b_{j}\right|+\left|b_{0}\right|\right)<+\infty$.

\section{Acknowledgment}

This research was partially supported by Waseda University grant for special research Projects 2006B-167 and Scientific Research (c), no. 19540229 of Japan Society for the Promotion of Science.

\section{References}

[1] Y. Song and C. T. H. Baker, "Perturbation theory for discrete Volterra equations," Journal of Difference Equations and Applications, vol. 9, no. 10, pp. 969-987, 2003.

[2] C. T. H. Baker and Y. Song, "Discrete Volterra operators, fixed point theorems and their application," Nonlinear Studies, vol. 10, no. 1, pp. 79-101, 2003.

[3] G. Gripenberg, S.-O. Londen, and O. Staffans, Volterra integral and functional equations, vol. 34 of Encyclopedia of Mathematics and Its Applications, Cambridge University Press, Cambridge, UK, 1990.

[4] M. S. Klamki, Ed., Problems in Applied Mathematics: Selections from SIAM Review, SIAM, Philadelphia, $\mathrm{Pa}, \mathrm{USA}, 1990$.

[5] M. R. Crisci, V. B. Kolmanovskii, E. Russo, and A. Vecchio, "Stability of difference Volterra equations: direct Liapunov method and numerical procedure," Computers $\mathcal{E}$ Mathematics with Applications, vol. 36, no. 10-12, pp. 77-97, 1998. 
[6] M. R. Crisci, V. B. Kolmanovskii, E. Russo, and A. Vecchio, "Stability of discrete Volterra equations of Hammerstein type," Journal of Difference Equations and Applications, vol. 6, no. 2, pp. 127-145, 2000.

[7] M. R. Crisci, V. B. Kolmanovskii, E. Russo, and A. Vecchio, "Asymptotic properties of solutions of Volterra difference equations with finite linear part," Dynamic Systems and Applications, vol. 8, no. 1, pp. 1-20, 1999.

[8] M. R. Crisci, V. B. Kolmanovskii, E. Russo, and A. Vecchio, "A priori bounds on the solution of a nonlinear Volterra discrete equation," Stability and Control: Theory and Applications, vol. 3, no. 1, pp. 38-47, 2000.

[9] S. N. Elaydi, V. L. Kocic, and J. Li, “Global stability of nonlinear delay difference equations," Journal of Difference Equations and Applications, vol. 2, no. 1, pp. 87-96, 1996.

[10] S. N. Elaydi, An Introduction to Difference Equations, Undergraduate Texts in Mathematics, Springer, New York, NY, USA, 3rd edition, 2005.

[11] Ch. Lubich, "On the numerical solution of Volterra equations with unbounded nonlinearity," Journal of Integral Equations, vol. 10, no. 1-3, pp. 175-183, 1985.

[12] A. Vecchio, "Boundedness of the global error of some linear and nonlinear methods for Volterra integral equations," Journal of Integral Equations and Applications, vol. 12, no. 4, pp. 449-465, 2000.

[13] M. R. Crisci, V. B. Kolmanovskii, E. Russo, and A. Vecchio, "Boundedness of discrete Volterra equations," Journal of Mathematical Analysis and Applications, vol. 211, no. 1, pp. 106-130, 1997.

[14] Ph. Clément and J. A. Nohel, "Asymptotic behavior of solutions of nonlinear Volterra equations with completely positive kernels," SIAM Journal on Mathematical Analysis, vol. 12, no. 4, pp. 514-535, 1981.

[15] A. Vecchio, "Volterra discrete equations: summability of the fundamental matrix," Numerische Mathematik, vol. 89, no. 4, pp. 783-794, 2001. 


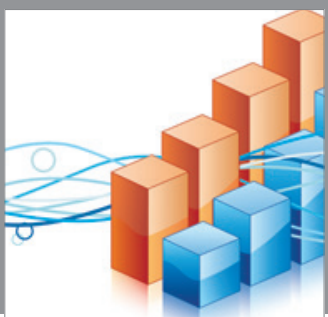

Advances in

Operations Research

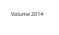

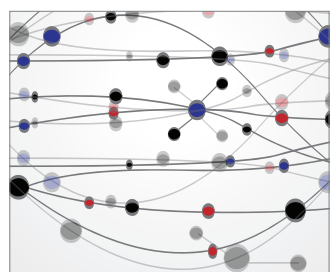

\section{The Scientific} World Journal
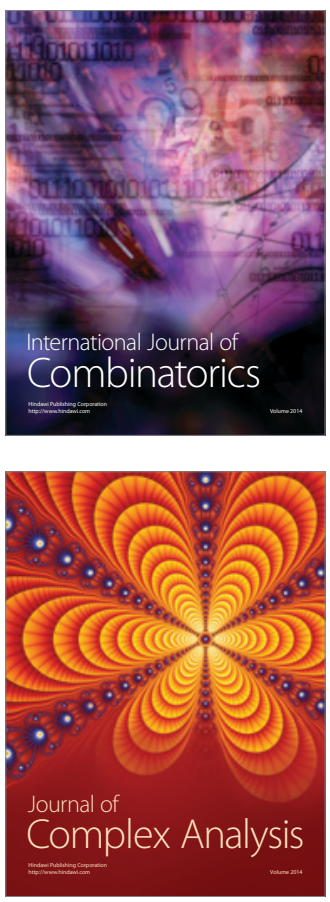

International Journal of

Mathematics and

Mathematical

Sciences
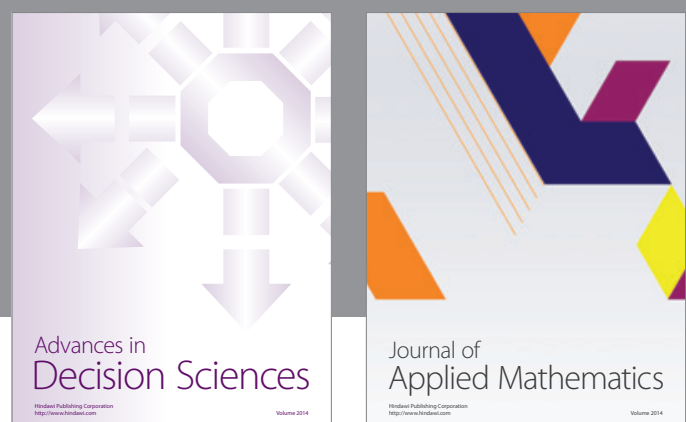

Journal of

Applied Mathematics
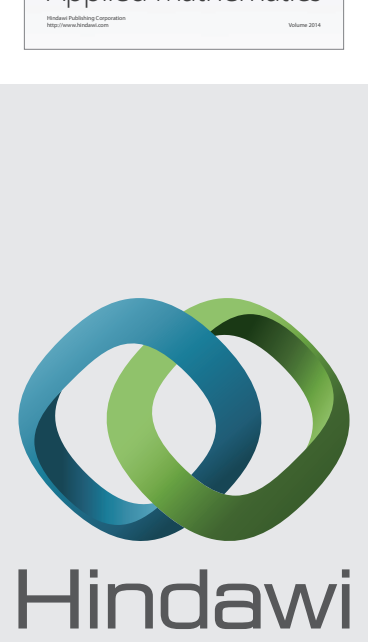

Submit your manuscripts at http://www.hindawi.com
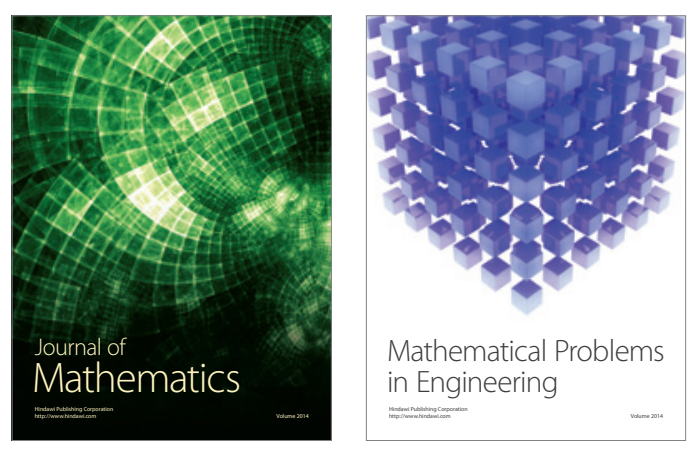

Mathematical Problems in Engineering
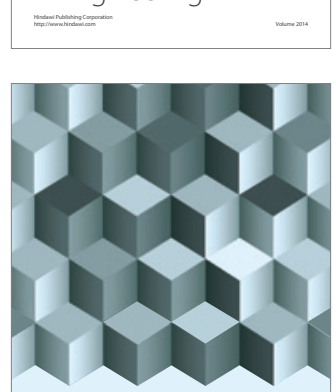

Journal of

Function Spaces
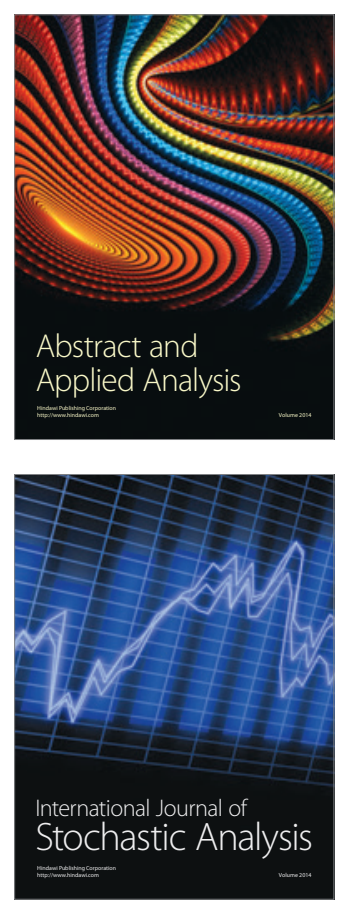

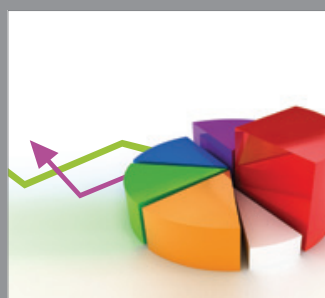

ournal of

Probability and Statistics

Promensencen
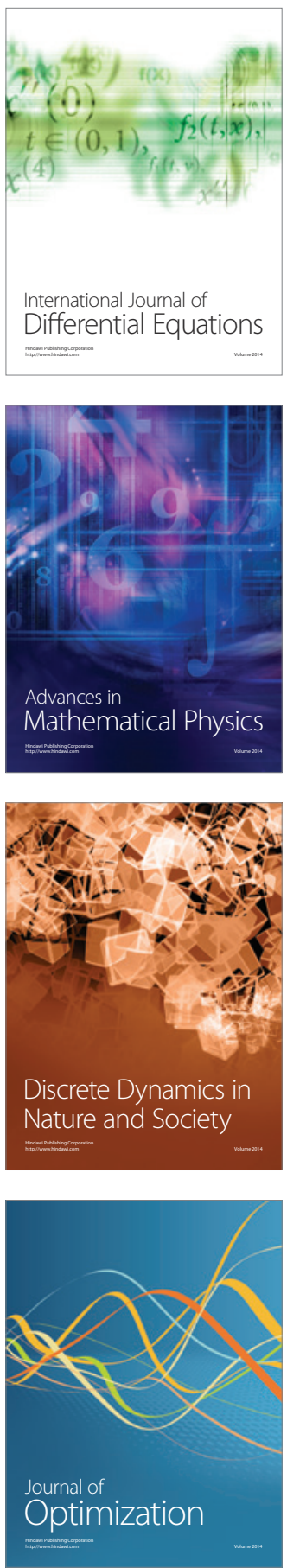